\title{
Improved lower bounds on partial lifetimes for nucleon decay modes
}

\author{
Sudhakantha Girmohanta $\odot$ and Robert Shrock $\odot$ \\ C. N. Yang Institute for Theoretical Physics and Department of Physics and Astronomy, \\ Stony Brook University, Stony Brook, New York 11794, USA
}

(Received 16 October 2019; published 16 December 2019)

\begin{abstract}
In the framework of a baryon-number-violating effective Lagrangian, we calculate improved lower bounds on partial lifetimes for proton and bound neutron decays, including $p \rightarrow \ell^{+} \ell^{\prime+} \ell^{\prime-}, n \rightarrow \bar{\nu} \ell^{+} \ell^{\prime-}$, $p \rightarrow \ell^{+} \nu \bar{\nu}$, and $n \rightarrow \bar{\nu} \bar{\nu} \nu$, where $\ell$ and $\ell^{\prime}$ denote $e$ or $\mu$, with both $\ell=\ell^{\prime}$ and $\ell \neq \ell^{\prime}$ cases. Our lower bounds are substantially stronger than the corresponding lower bounds from direct experimental searches. We also present lower bounds on $(\tau / B)_{p \rightarrow \ell^{+} \gamma},(\tau / B)_{n \rightarrow \bar{\nu} \gamma},(\tau / B)_{p \rightarrow \ell^{+} \gamma \gamma}$, and $(\tau / B)_{n \rightarrow \bar{\nu} \gamma \gamma}$. Our method relies on relating the rates for these decay modes to the rates for decay modes of the form $p \rightarrow \ell^{+} M$ and $n \rightarrow \bar{\nu} M$, where $M$ is a pseudoscalar or vector meson, and then using the experimental lower bounds on the partial lifetimes for these latter decays.
\end{abstract}

DOI: 10.1103/PhysRevD.100.115025

\section{INTRODUCTION}

Although the Standard Model (SM) conserves baryon number ${ }^{1} B$, this is violated in many of its ultraviolet extensions. This violation is natural in grand unified theories (GUTs) [2-5], since these theories place quarks and (anti)leptons in the same representation(s) of the GUT gauge group. More generally, baryon-number violation (BNV) is expected to occur in nature, because this is one of the necessary conditions for explaining the observed baryon asymmetry of the universe [6]. A number of dedicated experiments have been carried out since the early 1980s to search for proton decay and the decay of neutrons bound in nuclei. These experiments have obtained null results and have set resultant stringent upper limits for the rates of nucleon decays. ${ }^{2}$

In this paper, within the framework of a baryon-numberviolating effective Lagrangian, $\mathcal{L}_{\text {eff }}$, we shall calculate improved lower bounds on partial lifetimes for a number of nucleon decays. Let us denote the rate for the decay of a nucleon $N$ (where $N=p$ or $n$ ) to a final state $f . s$. as $\Gamma_{N \rightarrow f . s .}$, which is the inverse of the partial lifetime, $(\tau / B)_{N \rightarrow f . s .}=\left[\Gamma_{N \rightarrow f . s .}\right]^{-1}$, where $B$ denotes the branching

\footnotetext{
${ }^{1}$ Recall that the violation of $B$ by $\mathrm{SU}(2)_{L}$ instantons in the SM is negligibly small at temperatures low compared with the electroweak scale [1].

${ }^{2}$ We shall use the term "nucleon decay" to mean the decay of a proton or the $B$-violating decay of a neutron bound in a nucleus.

Published by the American Physical Society under the terms of the Creative Commons Attribution 4.0 International license. Further distribution of this work must maintain attribution to the author(s) and the published article's title, journal citation, and DOI. Funded by SCOAP ${ }^{3}$.
}

ratio for this decay mode. Our method is to derive an approximate relation between the rate $\Gamma_{N \rightarrow f . s .}$ for the decay of a nucleon to a final state f.s., $N \rightarrow f$.s. (where $N=p$ or $n$ ) and the rate for the decay $\Gamma_{N \rightarrow f . s . '}$ to a different final state, denoted f.s.'. Combining this relation with the experimental lower bound on $(\tau / B)_{N \rightarrow f . s .}$, we derive an approximate lower bound on $(\tau / B)_{N \rightarrow f . s .}$ for each final state f.s.'. Our theoretical framework is minimal, in the sense that the only physics beyond the SM (BSM) that is assumed is that which produces the basic set of local four-fermion operators in $\mathcal{L}_{\text {eff. }}$. If one were to assume the existence of other baryon-number-violating physics involving new particles with masses much smaller than the GUT scale, then other operators would become relevant, requiring a different analysis. Although the lower bounds that we derive are only approximate, they are useful because for many final states $f . s . '$ they are more stringent than the lower bounds on $(\tau / B)_{N \rightarrow f . s .}$ from direct experimental searches.

This paper is organized as follows. In Sec. II we discuss the four-fermion operators in $\mathcal{L}_{\text {eff }}$. We present our lower bounds on $(\tau / B)$ for $p \rightarrow \ell^{+} \gamma$ and $n \rightarrow \bar{\nu} \gamma$ in Sec. III. Sections IV and V contain our lower bounds on $(\tau / B)$ for $p \rightarrow \ell^{+} \ell^{\prime+} \ell^{\prime-}$ and $n \rightarrow \bar{\nu} \ell^{+} \ell^{-}$, where $\ell$ and $\ell^{\prime}$ denote $e$ or $\mu$, including both $\ell=\ell^{\prime}$ and $\ell \neq \ell^{\prime}$ cases. In Secs. VI and VII we derive lower bound bounds on $(\tau / B)$ for $p \rightarrow \ell^{+} \nu \bar{\nu}$ and $n \rightarrow \bar{\nu} \bar{\nu} \nu$. Here and below we use a symbolic notation in which $\nu$ may refer to an electroweak-doublet neutrino of some generation or to an electroweak-singlet neutrino; the context will make clear the meaning. In Sec. VIII we remark on the application of our method to other decays, including $p \rightarrow \ell^{+} \gamma \gamma$ and $n \rightarrow \bar{\nu} \gamma \gamma$. Our conclusions are presented in Sec. IX and some relevant phase-space formulas are given in the Appendix. 


\section{EFFECTIVE LAGRANGIAN}

Given the established experimental upper bounds on the rates for nucleon decays, it follows that the mass scale(s) characterizing the baryon-number-violating physics responsible for these days must be larger than the electroweak-symmetry-breaking scale, $v \simeq 250 \mathrm{GeV}$. Hence, one can analyze these decays using an effective Lagrangian, $\mathcal{L}_{\text {eff }}$, that is invariant not only with respect to color $\mathrm{SU}(3)_{c}$, but also with respect to gauge transformations of the electroweak gauge group, $G_{\mathrm{EW}}=\mathrm{SU}(2)_{L} \otimes$ $\mathrm{U}(1)_{Y}$. With the original SM fermions, before the addition of any electroweak-singlet neutrinos, the four-fermion operators $\mathcal{O}_{i}$ in $\mathcal{L}_{\text {eff }}$ that contribute to nucleon decays are as follows. We denote $Q_{a, L}^{\alpha}=\left(\begin{array}{l}Q_{a}^{1 \alpha} \\ Q_{a}^{2 \alpha}\end{array}\right)_{L}=\left(\begin{array}{l}u_{\alpha}^{\alpha} \\ d_{a}^{\alpha}\end{array}\right)_{L}$ and $L_{a, L}=\left(\begin{array}{c}L_{a}^{1} \\ L_{a}^{2}\end{array}\right)_{L}=\left(\begin{array}{c}\nu_{\ell_{a}} \\ \ell_{a}\end{array}\right)_{L}$, where $\alpha, \beta$, and $\gamma$ are $\mathrm{SU}(3)_{c}$ indices and $a$ is a generation index, with $d_{1}^{\alpha}=d^{\alpha}, d_{2}^{\alpha}=s^{\alpha}$, $d_{3}^{\alpha}=b^{\alpha}, \ell_{1}=e, \ell_{2}=\mu, \ell_{3}=\tau$, etc. The operators contributing to proton decay $\operatorname{are}^{3}[7,8]$

$$
\begin{gathered}
\mathcal{O}_{1}=\epsilon_{\alpha \beta \gamma}\left[u_{a_{1}, R}^{\alpha T} C d_{a_{2}, R}^{\beta}\right]\left[u_{a_{3}, R}^{\gamma T} C \ell_{a_{4}, R}\right], \\
\mathcal{O}_{2}=\epsilon_{i j} \epsilon_{\alpha \beta \gamma}\left[Q_{a_{1}, L}^{i \alpha T} C Q_{a_{2}, L}^{j \beta}\right]\left[u_{a_{3}, R}^{\gamma T} C \ell_{a_{4}, R}\right], \\
\mathcal{O}_{3}=\epsilon_{k m} \epsilon_{\alpha \beta \gamma}\left[u_{a_{1}, R}^{\alpha T} C d_{a_{2}, R}^{\beta}\right]\left[Q_{a_{3}, L}^{k \gamma T} C L_{a_{4}, L}^{m}\right],
\end{gathered}
$$

and

$$
\mathcal{O}_{4}=\epsilon_{i j} \epsilon_{k m} \epsilon_{\alpha \beta \gamma}\left[Q_{a_{1}, L}^{i \alpha T} C Q_{a_{2}, L}^{j \beta}\right]\left[Q_{a_{3}, L}^{k \gamma T} C L_{a_{4}, L}^{m}\right],
$$

where $C$ is the Dirac charge conjugation matrix satisfying $C \gamma_{\mu} C^{-1}=-\left(\gamma_{\mu}\right)^{T}, C=-C^{T}$ and $i, j, k$, and $m$ are $\mathrm{SU}(2)_{L}$ indices. As noted in [7], four-fermion operators with bilinears involving Dirac vector and tensor operators $\gamma_{\mu}$ and $\sigma_{\mu \nu}=(i / 2)\left[\gamma_{\mu}, \gamma_{\nu}\right]$ can be transformed to the operators listed above via Fierz identities. These operators have $\Delta B=-1$ and $\Delta L=-1$, where $L$ denotes total lepton number.

After the discovery of nonzero neutrino masses and lepton flavor mixing, a natural generalization of the Standard Model has involved the introduction of a set of electroweak-singlet neutrinos $\nu_{s, R}, s=1, \ldots, n_{s}$, which are necessary to form Dirac neutrino mass terms via Yukawa couplings $\sum_{a=1}^{3} \sum_{s=1}^{n_{s}} y_{a s}\left[\bar{L}_{a, L} \nu_{s, R} \tilde{\phi}\right]+$ H.c., where $\tilde{\phi} \equiv$ $i \sigma_{2} \phi^{*}$ and $\phi=\left(\begin{array}{c}\phi^{+} \\ \phi^{0}\end{array}\right)$ is the SM Higgs doublet. These $\nu_{s, R}$ neutrinos also generically form Majorana bare mass terms $\sum_{s, s^{\prime}=1}^{n_{s}} M_{s, s^{\prime}}^{(\nu)} \nu_{s, R}^{T} C \nu_{s^{\prime}, R}+$ H.c., thereby explicitly breaking

\footnotetext{
${ }^{3}$ Two other operators vanish identically in the case $a_{1}=a_{2}=$ $a_{3}=1$ relevant for nucleon decay, namely $\mathcal{O}_{5}=\epsilon_{\alpha \beta \gamma}\left[u_{a_{1}, R}^{\alpha T} C u_{a_{2}, R}^{\beta}\right]$ $\times\left[d_{a_{3}, R}^{\gamma T} C \ell_{a_{4}, R}\right]$ and $\mathcal{O}_{6}=\left(\epsilon_{i k} \epsilon_{j m}+\epsilon_{i m} \epsilon_{j k}\right) \epsilon_{\alpha \beta \gamma}\left[Q_{a_{1}, L}^{i \alpha T} C Q_{a_{2}, L}^{j \beta}\right] \times$ $\left[Q_{a_{3}, L}^{k \gamma T} C L_{a_{4}, L}^{m}\right]$.
}

total lepton number $L$ by 2 units. With the inclusion of these $\nu_{s, R}$, there are two additional types of operators for nucleon decay, namely

$$
\mathcal{O}_{7}=\epsilon_{\alpha \beta \gamma}\left[u_{a_{1}, R}^{\alpha T} C d_{a_{2}, R}^{\beta}\right]\left[d_{a_{3}, R}^{\gamma T} C \nu_{s, R}\right]
$$

and

$$
\mathcal{O}_{8}=\epsilon_{i j} \epsilon_{\alpha \beta \gamma}\left[Q_{a_{1}, L}^{i \alpha T} C Q_{a_{2}, L}^{j \beta}\right]\left[d_{a_{3}, R}^{\gamma T} C \nu_{s, R}\right]
$$

The generation indices $\left(a_{1}, a_{2}, a_{3}, a_{4}\right)$ in the $\mathcal{O}_{r}$ with $1 \leq r \leq 4$ and the indices $\left(a_{1}, a_{2}, a_{3}, s\right)$ in $\mathcal{O}_{r}$ with $r=7,8$ will be left implicit in the notation.

In terms of these fields, a minimal low-energy effective Lagrangian giving rise to nucleon decay can be written as

$$
\mathcal{L}_{\text {eff }}=\sum_{r} \sum_{\left\{a_{i}\right\} ; s} c_{r} \mathcal{O}_{r}
$$

where the second sum is over all of the generation indices $\left(a_{1}, a_{2}, a_{3}, a_{4}\right)$ in the operators $\mathcal{O}_{r}, 1 \leq r \leq 4$, and the indices $\left(a_{1}, a_{2}, a_{3}, s\right)$ in $\mathcal{O}_{7}$ and $\mathcal{O}_{8}$. Since these operators $\mathcal{O}_{r}$ have Maxwellian dimension 6 (in mass units), the coefficients $c_{r}$ have dimension -2 , and we write $c_{r}=\bar{c}_{r} /\left(M_{\mathrm{BNV}}\right)^{2}$, where $M_{\mathrm{BNV}}$ denotes an effective mass scale characterizing the baryon-number violation. In general, $\bar{c}_{r}$ depends on the generational indices of fermion fields in $\mathcal{O}_{r}$; this is again left implicit in the notation.

\section{THE DECAYS $p \rightarrow \ell^{+} \gamma$ AND $n \rightarrow \bar{\nu} \gamma$}

We begin by deriving approximate lower limits, within this theoretical framework, on the partial lifetimes for the decays $p \rightarrow \ell^{+} \gamma$ and $n \rightarrow \bar{\nu} \gamma$, where $\ell^{+}=e^{+}$or $\mu^{+}$and $\bar{\nu}$ may be an electroweak-nonsinglet antineutrino, $\bar{\nu}_{e}, \bar{\nu}_{\mu}$, or $\bar{\nu}_{\tau}$, or an electroweak-singlet antineutrino, $\bar{\nu}_{s}$, where $1 \leq s \leq n_{s}{ }^{4}$ In view of these possibilities, we omit a subscript on $\bar{\nu}$ here and in similar cases below. With our $\mathcal{L}_{\text {eff }}$, the leading contributions to the decay $p \rightarrow \ell^{+} \gamma$ arise from the diagrams in Fig. 1. Figure 1(a) shows a process in which $\sum_{r} c_{r} \mathcal{O}_{r}$ in $\mathcal{L}_{\text {eff }}$ (represented by the blob at the fourfermion vertex) with $1 \leq r \leq 4$ and $a_{1}=a_{2}=a_{3}=1$, $a_{4}=1$ or 2 for $\ell^{+}=e^{+}$or $\mu^{+}$, transforms an initial $u u$ pair in the proton to $\ell^{+} d^{c}$, and the $d^{c}$ annihilates with the $d$ in the proton to produce the outgoing photon. This figure is also understood to include a process in which the $d$ quark in the proton emits the photon, transitioning to a virtual $d$ that undergoes the BNV process depicted by the blob, resulting in the outgoing $\ell^{+}$. Figure 1(b) shows the analogous processes involving the $\mathrm{BNV}$ transformation

\footnotetext{
${ }^{4}$ In general, the EW-singlet interaction eigenstate $\bar{\nu}_{s}$ is a linear combination of mass eigenstates. Here the statement refers to the mass eigenstates with masses that are sufficiently small so that they are kinematically allowed to occur in the decay. This is also understood for the EW-nonsinglet $\bar{\nu}_{\ell}$.
} 


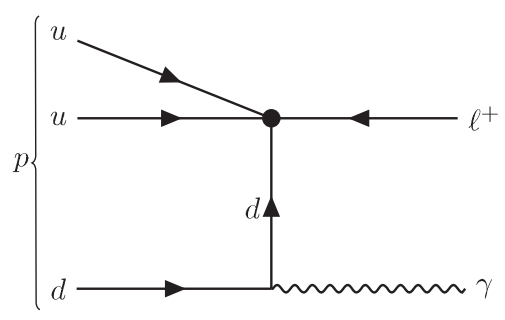

(a)

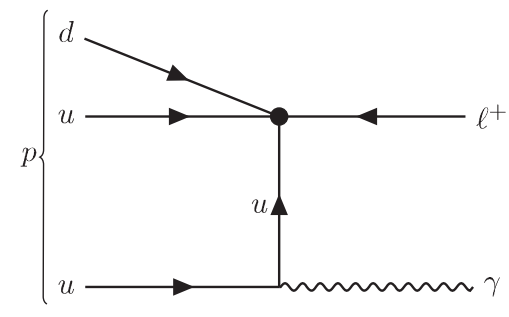

(b)

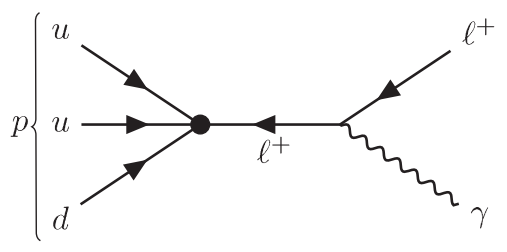

(c)

FIG. 1. Feynman diagrams for $p \rightarrow \ell^{+} \gamma$ with $\ell^{+}=e^{+}, \mu^{+}$.

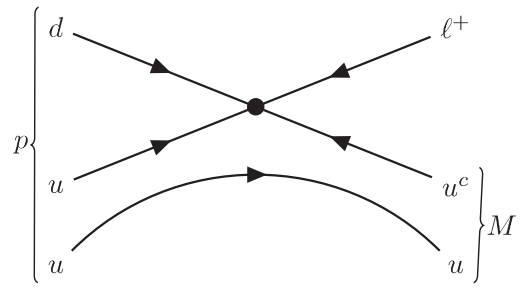

(a)

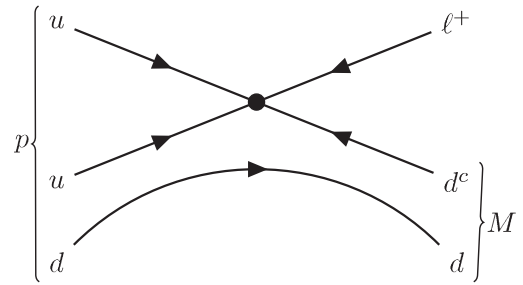

(b)

FIG. 2. Feynman diagrams for $p \rightarrow \ell^{+} M$, where $\ell^{+}=e^{+}, \mu^{+}$and $M$ denotes a pseudoscalar or vector meson.

$d u \rightarrow \ell^{+} u^{c}$. In Fig. 1(c), the initial uud quarks in the proton are transformed via the BNV $\mathcal{L}_{\text {eff }}$ to a virtual $s$-channel $\ell^{+}$that then radiates the photon.

To proceed, we relate the amplitude for the $p \rightarrow \ell^{+} \gamma$ decay to an amplitude for $p \rightarrow \ell^{+} M$ decay, where $\ell^{+}=e^{+}$ or $\mu^{+}$and $M$ denotes a neutral meson containing light quarks, such as $\pi^{0}, \eta, \rho^{0}$, or $\omega$. Feynman diagrams for the decay $p \rightarrow \ell^{+} M$ are shown in Fig. 2. Both the isoscalar mesons $\eta$ and $\omega$ and the isovector mesons $\pi^{0}$ and $\rho^{0}$ are relevant for this relation, since the electromagnetic current has both isoscalar and isovector parts, as embodied in the relation $Q_{\mathrm{em}}=I_{3}+(Y / 2)$, where here $I$ and $Y$ denote isospin and hypercharge, respectively. The final states involving the vector mesons $\omega$ and $\rho^{0}$ share with the final state involving the photon the property that they are all vector $(J=1)$ particles. On the other hand, the final states involving the $\pi^{0}$ and $\eta$ have (mass) ${ }^{2}$ values 0.0182 and $0.301 \mathrm{GeV}^{2}$ that are smaller than the (mass) ${ }^{2}$ values of 0.602 and $0.613 \mathrm{GeV}^{2}$ for the $\omega$ and $\rho^{0}$ and hence closer to the zero mass of the photon. In view of the complementary similarities [in spin and (mass) $)^{2}$ ] of the $\omega$ and $\rho^{0}$ hadronic final states, on the one hand, and the $\pi^{0}$ and $\eta$ hadronic final states, on the other, to the photon, we shall use all of these decay modes for our comparison. We list the experimental lower bounds on the partial lifetimes $(\tau / B)$ for relevant proton and bound neutron decay modes in Tables I and II. These and other limits listed here are at the $90 \%$ confidence level.

The phase-space factor for a decay of a nucleon $N$ to a two-body final state with particles of masses $m_{1}$ and $m_{2}$ is given by Eq. (A5) in the Appendix. We list the values of $(8 \pi) R_{2}^{(f . s .)}=\left[\lambda\left(1,\left(m_{1} / m_{N}\right)^{2},\left(m_{2} / m_{N}\right)^{2}\right]^{1 / 2}\right.$ in Table III for nucleon decays to various final states f.s. of relevance here.

Because the hadronic matrix elements $\left\langle M\left|\mathcal{L}_{\text {eff }}\right| p\right\rangle$ and $\left\langle 0\left|\mathcal{L}_{\text {eff }}\right| p\right\rangle$ that enter in the respective $p \rightarrow \ell^{+} M$ and $p \rightarrow \ell^{+} \gamma$ decays are different and the coefficients $c_{r}$ that enter into $\mathcal{L}_{\text {eff }}$ depend on the UV completion of the Standard Model that is responsible for the baryon-number violation, we will restrict our analysis to a rough estimate of the relation between the corresponding decays. (For lattice calculations of hadronic matrix elements, see Ref. [9].) A similar comment applies to $n \rightarrow \bar{\nu} \gamma$ decays. We have

$$
\Gamma_{p \rightarrow \ell^{+} \gamma} \sim e^{2}\left[\frac{R_{2}^{\left(\ell^{+} \gamma\right)}}{R_{2}^{\left(\ell^{+} M\right)}}\right] \Gamma_{p \rightarrow \ell^{+} M},
$$

where $\ell^{+}=e^{+}$or $\mu^{+}$and $e^{2}=4 \pi \alpha_{\mathrm{em}}$ is the squared electromagnetic coupling. The ratio of phase-space factors is included to take account of the difference in phase space for the $p \rightarrow \ell^{+} \gamma$ and $p \rightarrow \ell^{+} M$ decay modes. Equivalently,

$$
(\tau / B)_{p \rightarrow \ell^{+} \gamma} \sim\left(4 \pi \alpha_{\mathrm{em}}\right)^{-1}\left[\frac{R_{2}^{\left(\ell^{+} M\right)}}{R_{2}^{\left(\ell^{+} \gamma\right)}}\right](\tau / B)_{p \rightarrow \ell^{+} M} .
$$

Note that although the branching ratios for the various decay modes in Eq. (9) depend on the UV completion of the SM, the basic relation (8) is between the absolute rates themselves, which do not depend on these branching ratios.

We next make use of the experimental lower bounds on the partial lifetimes for various relevant proton decay modes, as displayed in Table I. These lower bounds on 
TABLE I. List of (a) experimental lower bounds (at the

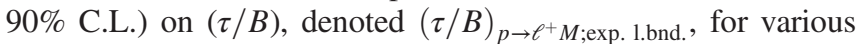
proton decays of the form $p \rightarrow \ell^{+} M$, where $\ell^{+}=e^{+}$or $\mu^{+}$and $M=\pi^{0}, \eta, \rho^{0}, \omega$, with references, given in the first to third columns; (b) in the fourth column, our resultant estimated lower bounds on $(\tau / B)$ for $p \rightarrow \ell^{+} \gamma$ obtained from Eq. (9), denoted $(\tau / B)_{p \rightarrow \ell^{+} \gamma \text {; est. 1.bnd. }}$ All limits on $(\tau / B)$ are given in units of $10^{33} \mathrm{yr}$. See the text for further discussion.

\begin{tabular}{lccc}
\hline \hline$p \rightarrow \ell^{+} M$ & $(\tau / B)_{p \rightarrow \ell^{+} M \text {;exp. 1.bnd. }}$ & Ref. & $(\tau / B)_{p \rightarrow \ell^{+} \gamma \text {;est. 1.bnd. }}$ \\
\hline$p \rightarrow e^{+} \pi^{0}$ & 16.0 & {$[10]$} & $2 \times 10^{2}$ \\
$p \rightarrow \mu^{+} \pi^{0}$ & 7.7 & {$[10]$} & $0.8 \times 10^{2}$ \\
$p \rightarrow e^{+} \eta$ & 10.0 & {$[11]$} & $0.7 \times 10^{2}$ \\
$p \rightarrow \mu^{+} \eta$ & 4.7 & {$[11]$} & 30 \\
$p \rightarrow e^{+} \rho^{0}$ & 0.720 & {$[11]$} & 2 \\
$p \rightarrow \mu^{+} \rho^{0}$ & 0.570 & {$[11]$} & 1 \\
$p \rightarrow e^{+} \omega$ & 1.60 & {$[11]$} & 5 \\
$p \rightarrow \mu^{+} \omega$ & 2.80 & {$[11]$} & 7 \\
\hline \hline
\end{tabular}

$(\tau / B)$ are all from the SuperKamiokande (SK) experiment; the bounds for $p \rightarrow \ell^{+} \pi^{0}$ and $p \rightarrow \ell^{+} \eta$ are from Ref. [10], while the bounds for $p \rightarrow \ell^{+} \omega$ and $p \rightarrow \ell^{+} \rho$ are from Ref. [11]. In the rightmost column of Table I, we list our estimates for the lower bounds on $p \rightarrow \ell^{+} \gamma$ obtained by combining the relation (9) with the experimental lower bounds on $(\tau / B)_{p \rightarrow \ell^{+} M}$ given in the middle column, for $\ell^{+}=e^{+}$and $\ell^{+}=\mu^{+}$. In view of the approximate nature of our estimated lower bounds on $(\tau / B)_{p \rightarrow \ell^{+} \gamma}$ in Table I, we list these only to one significant figure, and we follow this format with our estimates for other nucleon decay modes below.

Our estimates for lower bounds on $(\tau / B)_{p \rightarrow \ell^{+} \gamma}$ may be compared with lower bounds from direct experimental searches, which are as follows. The IMB-3 experiment obtained the limits [13] $(\tau / B)_{p \rightarrow e^{+} \gamma}>0.670 \times 10^{33} \mathrm{yr}$ and $(\tau / B)_{p \rightarrow \mu^{+} \gamma}>0.478 \times 10^{33} \mathrm{yr}$. More recently, the SK experiment has reported the limits [15] $(\tau / B)_{p \rightarrow e^{+} \gamma}>$ $4.1 \times 10^{34} \mathrm{yr}$ and $(\tau / B)_{p \rightarrow \mu^{+} \gamma}>2.4 \times 10^{34} \mathrm{yr}$. These comparisons are summarized in Table IV. In this table,

TABLE II. List of (a) experimental lower bounds (at the

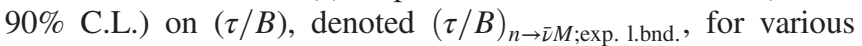
baryon-number-violating neutron decays of the form $n \rightarrow \bar{\nu} M$, where $M=\pi^{0}, \eta, \rho^{0}, \omega$, with references, given in the first to third columns; (b) in the fourth column, our resultant estimated lower bounds on $(\tau / B)$ for $n \rightarrow \bar{\nu} \gamma$ obtained from Eq. (10), denoted

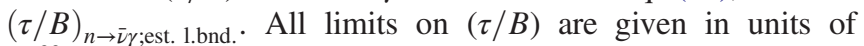
$10^{33} \mathrm{yr}$. See the text for further discussion.

\begin{tabular}{lccc}
\hline \hline$n \rightarrow \bar{\nu} M$ & $(\tau / B)_{n \rightarrow \bar{\nu} M \text {;exp. 1.bnd. }}$ & Ref. & $(\tau / B)_{n \rightarrow \bar{\nu} \gamma \text {; est. 1.bnd. }}$ \\
\hline$n \rightarrow \bar{\nu} \pi^{0}$ & 1.1 & {$[12]$} & 10 \\
$n \rightarrow \bar{\nu} \eta$ & 0.158 & {$[13]$} & 1 \\
$n \rightarrow \bar{\nu} \omega$ & 0.108 & {$[13]$} & 0.4 \\
$n \rightarrow \bar{\nu} \rho^{0}$ & $1.9 \times 10^{-2}$ & {$[14]$} & 0.07 \\
\hline \hline
\end{tabular}

TABLE III. Reduced two-body phase-space factors $(8 \pi) R_{2}^{(f . s .)}$ for two-body proton decays to the indicated final states ( $f . s$.).

\begin{tabular}{lc}
\hline \hline Decay & $(8 \pi) R_{2}^{(f . s .)}$ \\
\hline$p \rightarrow e^{+} \gamma$ & 1.000 \\
$p \rightarrow \mu^{+} \gamma$ & 0.987 \\
$p \rightarrow e^{+} \pi^{0}$ & 0.979 \\
$p \rightarrow \mu^{+} \pi^{0}$ & 0.966 \\
$p \rightarrow e^{+} \eta$ & 0.658 \\
$p \rightarrow \mu^{+} \eta$ & 0.632 \\
$p \rightarrow e^{+} \rho^{0}$ & 0.316 \\
$p \rightarrow \mu^{+} \rho^{0}$ & 0.241 \\
$p \rightarrow e^{+} \omega$ & 0.304 \\
$p \rightarrow \mu^{+} \omega$ & 0.222 \\
$n \rightarrow \bar{\nu} \gamma$ & 1.000 \\
$n \rightarrow \bar{\nu} \pi^{0}$ & 0.979 \\
$n \rightarrow \bar{\nu} \eta$ & 0.659 \\
$n \rightarrow \bar{\nu} \rho^{0}$ & 0.318 \\
$n \rightarrow \bar{\nu} \omega$ & 0.306 \\
\hline \hline
\end{tabular}

for each of the decay modes $p \rightarrow \ell^{+} \gamma$ and $n \rightarrow \bar{\nu} \gamma$, we list the range of estimated lower bounds that we obtain using Eq. (9) with all of the input bounds for $p \rightarrow \ell^{+} M$.

We proceed to carry out the corresponding analysis for the decay $n \rightarrow \bar{\nu} \gamma$. The leading Feynman diagrams contributing to this decay are shown in Fig. 3. As noted above, since the antineutrino is not observed, it could either be an electroweak-nonsinglet, $\bar{\nu}_{\ell}$ (i.e., $\bar{\nu}_{e}, \bar{\nu}_{\mu}$, or $\bar{\nu}_{\tau}$ ) or an EWsinglet, $\bar{\nu}_{s}$. The leading contributions to the $n \rightarrow \bar{\nu}_{\ell} \gamma$ decay arise from the relevant terms in $\mathcal{O}_{3}$ and $\mathcal{O}_{4}$ with $a_{1}=a_{2}=$ $a_{3}=1$ and arbitrary $a_{4}$ (where $a_{4}=1,2,3$ for $\bar{\nu}_{e}, \bar{\nu}_{\mu}$,

TABLE IV. Table listing (a) experimental lower bounds (l.bnd.) (at the $90 \%$ C.L.) on $(\tau / B)$ for various nucleon decays, denoted

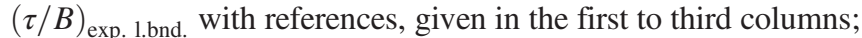
and (b) our theoretical estimated lower bounds on the partial lifetimes for these nucleon decays, denoted $(\tau / B)_{\text {est. 1.bnd. The }}$ units of $(\tau / B)$ are $10^{33} \mathrm{yr}$. The abbreviation NA means "not available." See the text for further details.

\begin{tabular}{lccc}
\hline \hline Decay mode & $(\tau / B)_{\text {exp. 1.bnd. }}$ & Ref. & $(\tau / B)_{p \rightarrow \ell^{+} \gamma \text {;est. 1.bnd. }}$ \\
\hline$p \rightarrow e^{+} \gamma$ & 41 & {$[15]$} & $\sim 10-10^{2}$ \\
$p \rightarrow \mu^{+} \gamma$ & 24 & {$[15]$} & $\sim 10-10^{2}$ \\
$p \rightarrow e^{+} \gamma \gamma$ & 1.00 & {$[16]$} & $\sim 10^{4}$ \\
$p \rightarrow \mu^{+} \gamma \gamma$ & NA & NA & $\sim 10^{4}$ \\
$n \rightarrow \bar{\nu} \gamma$ & 0.55 & {$[17]$} & $\sim 1-10$ \\
$n \rightarrow \bar{\nu} \gamma \gamma$ & 2.19 & {$[13]$} & $\sim 10^{3}$ \\
$p \rightarrow e^{+} e^{+} e^{-}$ & 0.793 & {$[13]$} & $\sim 10^{4}$ \\
$p \rightarrow \mu^{+} e^{+} e^{-}$ & 0.529 & {$[13]$} & $\sim 10^{4}$ \\
$p \rightarrow e^{+} \mu^{+} \mu^{-}$ & 0.359 & {$[13]$} & $\sim 10^{4}$ \\
$p \rightarrow \mu^{+} \mu^{+} \mu^{-}$ & 0.675 & {$[13]$} & $\sim 10^{4}$ \\
$n \rightarrow \bar{\nu} e^{+} e^{-}$ & 0.257 & {$[13]$} & $\sim 10^{3}$ \\
$n \rightarrow \bar{\nu} \mu^{+} \mu^{-}$ & 0.079 & {$[13]$} & $\sim 10^{3}$ \\
$n \rightarrow \bar{\nu} \mu^{+} e^{-}$ & 0.083 & {$[13]$} & $\sim 10^{11}$ \\
$p \rightarrow \bar{\nu} e^{+} \nu_{e}$ & 0.17 & {$[18]$} & $\sim 10^{12}$ \\
$p \rightarrow \bar{\nu} \mu^{+} \nu_{\mu}$ & 0.22 & {$[18]$} & $\sim 10^{12}$ \\
$n \rightarrow \bar{\nu} \nu \bar{\nu}$ & $0.58 \times 10^{-3}$ & {$[19]$} & $\sim 10^{11}$ \\
\hline \hline
\end{tabular}




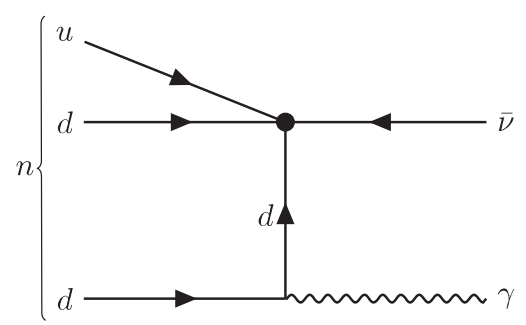

(a)

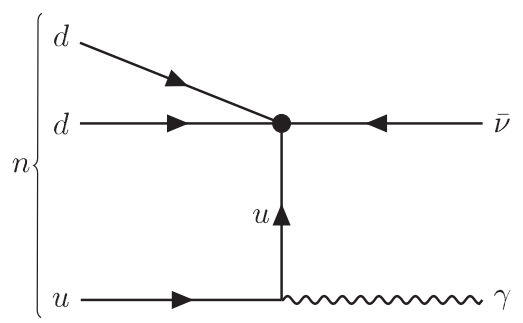

(b)

FIG. 3. Feynman diagrams for $n \rightarrow \bar{\nu} \gamma$.

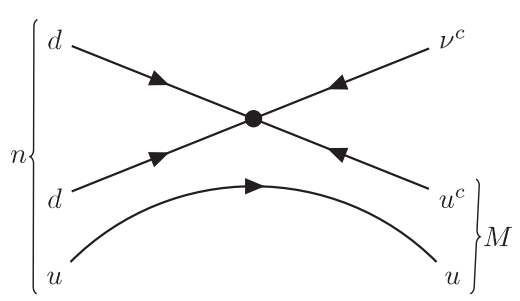

(a)

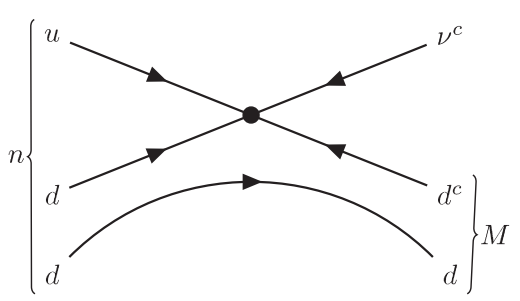

(b)

FIG. 4. Feynman diagrams for $n \rightarrow \bar{\nu} M$, where $M$ denotes a pseudoscalar or vector meson.

and $\bar{\nu}_{\tau}$ ), namely the term $-\epsilon_{\alpha \beta \gamma}\left[u_{R}^{\alpha T} C d_{R}^{\beta}\right]\left[d_{L}^{\gamma T} C \nu_{a_{4}, L}\right]$ in $\mathcal{O}_{3}$ and the term $-2 \epsilon_{\alpha \beta \gamma}\left[u_{L}^{\alpha}{ }^{T} C d_{L}^{\beta}\right]\left[d_{L}^{\gamma} C \nu_{a_{4}, L}\right]$ in $\mathcal{O}_{4}$. The leading contributions to $n \rightarrow \bar{\nu}_{s} \gamma$ arise from the term $\epsilon_{\alpha \beta \gamma}\left[u_{R}^{\alpha}{ }^{T} C d_{R}^{\beta}\right]\left[d_{R}^{\gamma T} C \nu_{s, R}\right]$ in $\mathcal{O}_{7}$ and the term $2\left[u_{L}^{\alpha}{ }^{T} C d_{L}^{\beta}\right]$ $\left[d_{R}^{\prime T} C \nu_{s, R}\right]$ in $\mathcal{O}_{8}$. Our procedure is again to obtain an approximate relation between the rates for $n \rightarrow \bar{\nu} \gamma$ and for $n \rightarrow \bar{\nu} M$. In Figs. 4(a) and 4(b), we show diagrams contributing to the decay $n \rightarrow \bar{\nu} M$. By the same method as we used above, we obtain the approximate estimate

$$
\begin{aligned}
(\tau / B)_{n \rightarrow \bar{\nu} \gamma} & \sim\left(4 \pi \alpha_{\mathrm{em}}\right)^{-1}\left[\frac{R_{2}^{(\bar{\nu} M)}}{R_{2}^{(\bar{\nu} \gamma)}}\right](\tau / B)_{n \rightarrow \bar{\nu} M} \\
& \sim\left(4 \pi \alpha_{\mathrm{em}}\right)^{-1}\left[1-\frac{m_{M}^{2}}{m_{n}^{2}}\right](\tau / B)_{n \rightarrow \bar{\nu} M} .
\end{aligned}
$$

We make use of the experimental lower bounds on $(\tau / B)$ for relevant decays $n \rightarrow \bar{\nu} M$, displayed in Table II. ${ }^{5}$ In the rightmost column of Table II, we list our estimates for the lower bounds on $n \rightarrow \bar{\nu} \gamma$ obtained by combining the relation (10) with the experimental lower bounds given in the middle column. As is again evident from this table, our approximate lower bounds on $(\tau / B)_{n \rightarrow \bar{\nu} \gamma}$ using the experimental limits on $n \rightarrow \bar{\nu} \pi^{0}$ and $n \rightarrow \bar{\nu} \eta$ are stronger than

\footnotetext{
${ }^{5}$ Since the experiments do not observe the $\bar{\nu}$, these experimental bounds are more general. For example, the lower bound on the partial lifetime for $n \rightarrow \bar{\nu} \pi^{0}$ actually applies to any decay of the form $n \rightarrow x^{0} \pi^{0}$, where $x^{0}$ is a neutral, weakly interacting particle or antiparticle that does not decay in the detector, and similarly for the other decay modes $n \rightarrow x^{0} M$. These subsume the case where $x^{0}=\bar{\nu}$ or $x^{0}=\nu$.
}

the bound from a direct experimental search, which is $(\tau / B)_{n \rightarrow \bar{\nu} \gamma}>0.550 \times 10^{33} \mathrm{yr}[17]$.

\section{IV. $p \rightarrow \ell^{+} \ell^{\prime+} \ell^{\prime-}$ DECAYS}

In this section we calculate estimated lower bounds on the partial lifetimes of several proton decays of the form $p \rightarrow \ell^{+} \ell^{\prime+} \ell^{\prime-}$, where $\ell$ and $\ell^{\prime}$ denote $e$ or $\mu$, including both of the cases $\ell=\ell^{\prime}$ and $\ell \neq \ell^{\prime}$. Graphs for the abovementioned decays are shown in Fig. 5. As discussed above, our theoretical framework for this and our other estimates is a minimal one in which we assume only the baryon-numberviolating physics beyond the Standard Model that gives rise to $\mathcal{L}_{\text {eff }}$. If one were to assume other BSM physics involving new particles with masses much smaller than the GUT scale, then other graphs and operators would become relevant (e.g., [20]). We denote the four-momenta of the $p, \ell^{+}, \ell^{\prime-}$, and $\ell^{\prime+}$ as $p, p_{3}, p_{2}$, and $p_{1}$, respectively, and set $q=p_{1}+p_{2}=p-p_{3}$. Let us first consider the $q \bar{q}$ annihilation producing a virtual photon, as indicated in Figs. 5(a) and 5(b). If the total angular momentum of the $q \bar{q}$ system is $J=0$, then the matrix element for this subprocess is proportional to $q^{\lambda}\left[\bar{u}\left(p_{2}\right) \gamma_{\lambda} v\left(p_{1}\right)\right]$ [where $\bar{u}\left(p_{2}\right)$ and $v\left(p_{1}\right)$ are Dirac spinors], which vanishes. In the corresponding terms involving $Z$ exchange, if the angular momentum $J$ of the $q \bar{q}$ subsystem is zero, then the matrix element for the subprocess is proportional to $q^{\lambda}\left[\bar{u}\left(p_{2}\right) \gamma_{\lambda}\right.$ $\left.\left\{-(1 / 2) P_{L}+\sin ^{2} \theta_{W}\right\} v\left(p_{1}\right)\right]$, where $P_{L}=\left(1-\gamma_{5}\right) / 2$. In this case, the vector part of the neutral current gives zero contribution and the axial-vector part gives a small term $\propto 2 m_{\ell^{\prime}}\left[\bar{u}\left(p_{2}\right) \gamma_{5} v\left(p_{1}\right)\right]$. Thus, the dominant contributions from these graphs are expected to arise from $q \bar{q}$ configurations with $J=1$. For these $J=1$ terms, the 


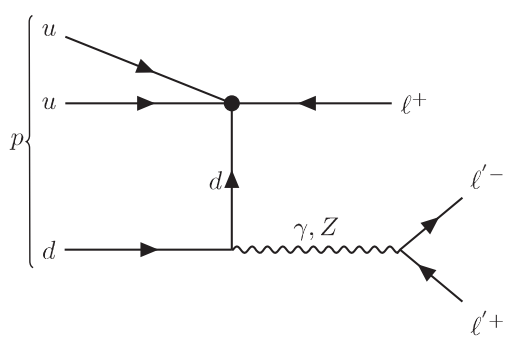

(a)

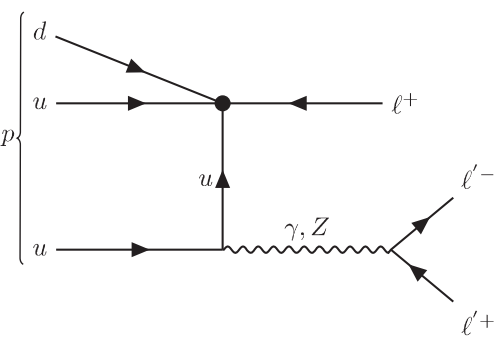

(b)

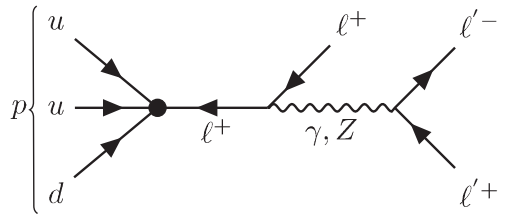

(c)

FIG. 5. Feynman diagrams for $p \rightarrow \ell^{+} \ell^{\prime+} \ell^{\prime-}$ with $\ell, \ell^{\prime}=e, \mu$.

contributions to the amplitude from the graphs with a virtual photon are expected to dominate over the contributions from the graphs with a virtual $Z$ by a factor $\sim e^{2} /\left(G_{F} m_{p}^{2}\right) \sim 10^{3}$. The contribution from the virtual photon in the graph of Fig. 5(c) is similarly dominant over that from the virtual $Z$. It is thus natural to use, for comparison, proton decays to vector mesons, $p \rightarrow e^{+} \rho^{0}$ and $p \rightarrow e^{+} \omega$. In contrast to the case with the $p \rightarrow \ell^{+} \gamma$ decays, this comparison connects a decay to a two-body final state to a decay with a three-body final state. Because the integral (A1) for the decay into a three-body final state involves details of $\mathcal{L}_{\text {eff }}$ in a nontrivial integration, while the corresponding integral for the decay into a two-body final state only involves a trivial angular integration (since the magnitudes of the three-momenta of the two final-state particles are fixed), it is difficult to make a precise comparison between the rates for these two decays. For a rough approximation, we will simply take into account the differences in phase space for these decays, via the ratios of the two-body and three-body phase-space factors. Since the three-body phase-space factor has dimensions of (mass) $)^{2}$ and the mass scale is set by the initial nucleon mass, we will introduce a dimensionless three-body phase-space factor for a decay to a given final state f.s., denoted $\bar{R}_{3}^{(f . s .)}$, as defined in Eq. (A4) in the Appendix. This quantity has the value $1 /\left(2^{8} \pi^{3}\right)$ if all of the three final-state particles have zero or negligibly small masses. Expressions for $\left(2^{8} \pi^{3}\right) \bar{R}_{3}^{(f . s .)}$ for relevant final states with non-negligible masses are given in Eqs. (A11)-(A13) in the Appendix.

We are thus led to the estimate

$$
\Gamma_{p \rightarrow \ell^{+} \ell^{\prime+} \ell^{--}} \sim\left(4 \pi \alpha_{\mathrm{em}}\right)^{2}\left[\frac{\bar{R}_{3}^{\left(\ell^{+} \ell^{\prime+} \ell^{\prime-}\right)}}{R_{2}^{\left(\ell^{+} M\right)}}\right] \Gamma_{p \rightarrow \ell^{+} M}
$$

or equivalently,

$$
(\tau / B)_{p \rightarrow \ell^{+} \ell^{\prime+} \ell^{\prime-}} \sim\left(4 \pi \alpha_{\mathrm{em}}\right)^{-2}\left[\frac{R_{2}^{\left(\ell^{+} M\right)}}{\bar{R}_{3}^{\left(\ell^{+} \ell^{\prime+} \ell^{\prime-}\right)}}\right](\tau / B)_{p \rightarrow \ell^{+} M} .
$$

Substituting the experimental lower bounds $(\tau / B)_{p \rightarrow e^{+} \rho^{0}}>$ $0.720 \times 10^{33} \mathrm{yr}$ and $(\tau / B)_{p \rightarrow e^{+} \omega}>1.60 \times 10^{33} \mathrm{yr}[11]$ in Eq. (12) and taking account of the ratios of phase-space factors, we obtain estimated lower bounds on $(\tau / B)_{p \rightarrow e^{+} e^{+} e^{-}}$ of $0.9 \times 10^{37}$ and $2 \times 10^{37} \mathrm{yr}$, respectively. These are much stronger than the lower bound on the partial lifetime for this decay from a direct experimental search, which is [13] $(\tau / B)_{p \rightarrow e^{+} e^{+} e^{-}}>0.793 \times 10^{33}$. Substituting the experimental lower bounds $(\tau / B)_{p \rightarrow \mu^{+} \rho^{0}}>0.570 \times 10^{33} \mathrm{yr}$ and $(\tau / B)_{p \rightarrow \mu^{+} \omega}>2.80 \times 10^{33} \mathrm{yr}[11]$ in Eq. (12) and computing the ratios of phase-space factors, we obtain estimated lower bounds on $(\tau / B)_{p \rightarrow \mu^{+} e^{+} e^{-}}$of $0.6 \times 10^{37}$ and $3 \times 10^{37} \mathrm{yr}$, respectively. We conservatively list these as $(\tau / B)_{p \rightarrow \mu^{+} e^{+} e^{-}} \gtrsim 10^{37} \mathrm{yr}$ in Table IV. Again, these are much stronger than the experimental lower bounds from direct searches, namely $(\tau / B)_{p \rightarrow \mu^{+} e^{+} e^{-}}>0.529 \times 10^{33}$ and $(\tau / B)_{p \rightarrow \mu^{+} \mu^{+} \mu^{-}}>0.675 \times 10^{33}$ [13]. These estimated lower bounds on partial lifetimes are summarized in Table IV, in comparison with the current lower bounds from direct experimental searches.

With regard to these and other nucleon decay modes for which our estimates yield lower bounds on the partial lifetimes that are much greater than existing bounds from direct experimental searches, we stress that this does not mean that these decay modes are not worth searching for in further experiments. If, for example, in the future, the decays $p \rightarrow e^{+} \pi^{0}$ and $p \rightarrow e^{+} e^{+} e^{-}$are both observed and the value of $(\tau / B)_{p \rightarrow e^{+} e^{+} e^{-}}$is significantly lower than a range estimated from Eq. (12), this would be doubly interesting, as evidence not only of baryon-number violation incorporated in $\mathcal{L}_{\text {eff }}$, but also of additional relevant physics beyond the Standard Model. This comment also applies for the other nucleon decay channels to be discussed below, for which our lower bounds on partial lifetimes are much higher than the lower bounds from direct experimental searches.

\section{V. $n \rightarrow \bar{\nu} \ell^{+} \ell^{-}$AND $n \rightarrow \bar{\nu} \ell^{+} \ell^{\prime-}$ DECAYS}

In this section we analyze the neutron decays $n \rightarrow \bar{\nu} \ell^{+} \ell^{-}$and $n \rightarrow \bar{\nu} \ell^{+} \ell^{\prime-}$, where $\ell, \ell^{\prime}=e, \mu$. Feynman diagrams for $n \rightarrow \bar{\nu} \ell^{+} \ell^{-}$decays are shown in Fig. 6. In the graphs in Figs. 6(a) and 6(b), the $\bar{\nu}$ could be an EW-doublet or an EW-singlet antineutrino, while in the graphs of Figs. 6(c) and 6(d), the $\bar{\nu}=\bar{\nu}_{\ell}$ is an EW-doublet antineutrino. Since an experiment would not observe the $\bar{\nu}$, 


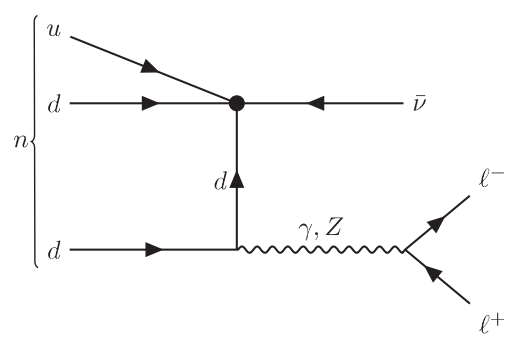

(a)

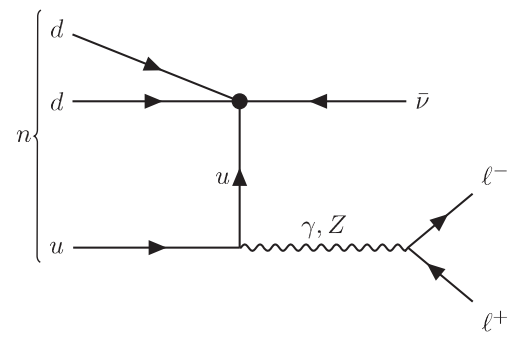

(b)

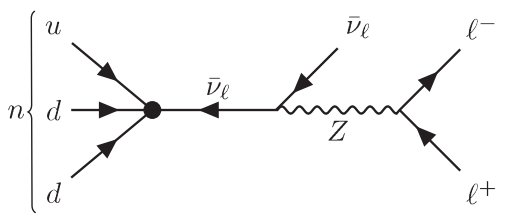

(c)

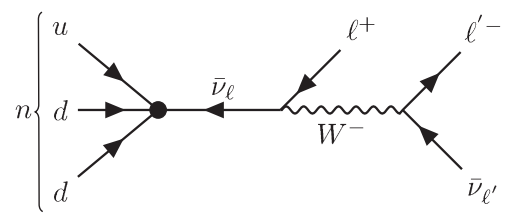

(d)

FIG. 6. Feynman diagrams for $n \rightarrow \bar{\nu} \ell^{+} \ell^{-}$and $n \rightarrow \bar{\nu} \ell^{+} \ell^{\prime-}$ with $\ell, \ell^{\prime}=e, \mu$.

it would not distinguish between these possibilities. In the graphs of Figs. 6(a)-6(c), the charged (anti)leptons are of the same generation, while in Fig. 6(d), $\ell^{\prime-}$ may be of a different generation than $\ell^{+}$. As discussed before, the contributions of the diagrams with a virtual $Z$ in Figs. 6(a)-6(c) are very small compared with the contributions of the diagrams of Figs. 6(a) and 6(b) with a virtual photon. This is also true of the graph with a virtual $W$ in Fig. 6(d). Therefore, by arguments similar to those used for the analysis of $p \rightarrow \ell^{+} \ell^{\prime+} \ell^{\prime-}$ decays, we estimate

$(\tau / B)_{n \rightarrow \bar{\nu} \ell^{+} \ell^{-}} \sim\left(4 \pi \alpha_{\mathrm{em}}\right)^{-2}\left[\frac{R_{2}^{(\bar{\nu} M)}}{\bar{R}_{3}^{\left(\bar{\nu} \ell^{+} \ell^{\prime-}\right)}}\right](\tau / B)_{n \rightarrow \bar{\nu} M}$.

For the same reasons discussed in connection with the $p \rightarrow$ $\ell^{+} \ell^{\prime+} \ell^{\prime-}$ decays, when applying the relation (13), we will use $n \rightarrow \bar{\nu} M$ decays with $M$ being a vector meson, $\rho^{0}$ or $\omega$. As an illustrative example, we consider the decay $n \rightarrow \bar{\nu} e^{+} e^{-}$. Since $m_{e}^{2} / m_{n}^{2}=3.0 \times 10^{-7}$ is negligibly small, it follows that, to very good accuracy, $\bar{R}_{3}=\bar{R}_{3,0}$ [see Eq. (A10)]. Therefore, Eq. (13) takes the explicit form

$$
\begin{aligned}
(\tau / B)_{n \rightarrow \bar{\nu} e^{+} e^{-}} & \sim\left(4 \pi \alpha_{\mathrm{em}}\right)^{-2}\left[\frac{R_{2}^{(\bar{\nu} M)}}{\left.\bar{R}_{3}^{\left(\bar{\nu} e^{+} e^{-}\right)}\right](\tau / B)_{n \rightarrow \bar{\nu} M}}\right. \\
& \sim\left(4 \pi \alpha_{\mathrm{em}}\right)^{-2}\left[\left(2^{5} \pi^{2}\right)\left(1-\frac{m_{M}^{2}}{m_{n}^{2}}\right)\right](\tau / B)_{n \rightarrow \bar{\nu} M} .
\end{aligned}
$$

Of the two $n \rightarrow \bar{\nu} M$ decay channels, the experimental lower bound on the channel with $M=\omega$ is the stronger one, so we focus on it. Substituting the lower bound $(\tau / B)_{n \rightarrow \bar{\nu} \omega}>1.08 \times 10^{32}$ from Ref. [13] in Eq. (13) and evaluating Eq. (14), we obtain the estimated lower bound $(\tau / B)_{n \rightarrow \bar{\nu} e^{+} e^{-}} \gtrsim 1.2 \times 10^{36} \mathrm{yr}$. This is much stronger than the lower bound from a direct search, namely $(\tau / B)_{n \rightarrow \bar{\nu} e^{+} e^{-}}>2.57 \times 10^{32} \mathrm{yr}$.

Next, again using the same experimental lower bound on $(\tau / B)_{n \rightarrow \bar{\nu} \omega}$ and computing the ratio of phase-space factors using Eqs. (A5) and (A13) in the Appendix, we obtain the estimated lower bound $(\tau / B)_{n \rightarrow \bar{\nu} \mu^{+} \mu^{-}} \gtrsim 1.6 \times 10^{36} \mathrm{yr}$. This is a much more stringent lower bound than the one from a direct experimental search, which is $(\tau / B)_{n \rightarrow \bar{\nu} \mu^{+} \mu^{-}}>$ $0.79 \times 10^{32} \mathrm{yr}$.

Finally, we discuss the decay $n \rightarrow \bar{\nu} e^{ \pm} \mu^{\mp}$. Only the Feynman diagram of Fig. 6(d) contributes to this decay, so we obtain the estimate

$$
(\tau / B)_{n \rightarrow \bar{\nu} e^{ \pm} \mu^{\mp}} \sim\left(G_{F} m_{n}^{2}\right)^{-2}\left[\frac{R_{2}^{(\bar{\nu} M)}}{\bar{R}_{3}^{\left(\bar{\nu} e^{ \pm} \mu^{\mp}\right)}}\right](\tau / B)_{n \rightarrow \bar{\nu} M} .
$$

Using the experimental lower bound on $(\tau / B)_{n \rightarrow \bar{\nu} \omega}$ again, we obtain the estimate $(\tau / B)_{n \rightarrow \bar{\nu} e^{ \pm} \mu^{\mp}} \gtrsim 1 \times 10^{44} \mathrm{yr}$. This is much stronger than the bound from the direct search for a decay of this type, namely $(\tau / B)_{n \rightarrow \bar{\nu} \mu^{+}} e^{-}>0.83 \times$ $10^{32} \mathrm{yr}[13]$.

$$
\text { VI. } p \rightarrow \ell^{+} \nu \bar{\nu}
$$

In this section we derive an estimated bound for several different types of proton decays which are experimentally indistinguishable, namely (i) $p \rightarrow \ell^{+} \nu_{\ell^{\prime}} \overline{\boldsymbol{\nu}}_{\ell^{\prime}}$; (ii) $p \rightarrow \ell^{+} \nu_{\ell} \bar{\nu}_{\ell^{\prime}}$; and (iii) $p \rightarrow \ell^{+} \nu_{\ell} \bar{\nu}_{s}$. We will refer collectively to these as $p \rightarrow \ell^{+} \nu \bar{\nu}$. Experimentally, these are all of the form $p \rightarrow \ell^{+}+$missing, where "missing" denotes two neutral weakly interacting particles, antiparticles, or a particle-antiparticle pair, which do not decay in the detector. Experimental papers often use the symbolic notation $p \rightarrow \ell^{+} \nu \nu$ for all of these decays. Graphs that contribute to the decays (i)-(iii) are shown in Fig. 7. The 


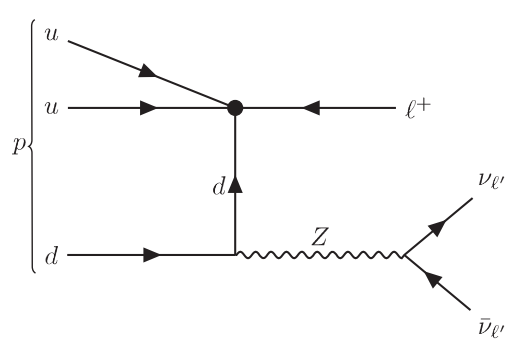

(a)

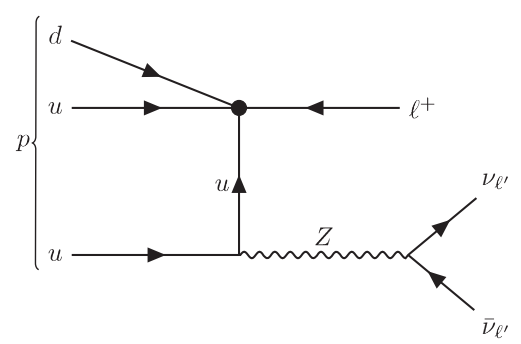

(b)

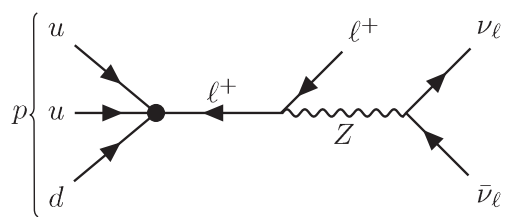

(c)

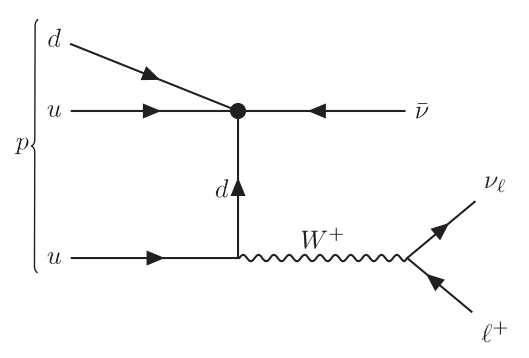

(d)

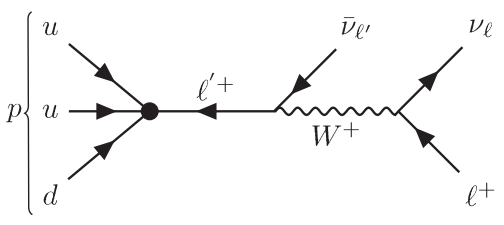

(e)

FIG. 7. Feynman diagrams for $p \rightarrow \ell^{+} \nu \bar{\nu}$ with $\ell^{+}=e^{+}, \mu^{+}$.

graphs in Figs. 7(a)-7(c) contain an internal $Z$ line and contribute to decays of type (i) and the subset of the decays of type (ii) in which $\nu_{\ell}=\nu_{\ell^{\prime}}$. The graphs in Figs. 7(d) and 7(e) contain an internal $W$ line and contribute to decays of the form (ii) and (iii), depending on whether the $\bar{\nu}$ emitted from the effective four-fermion BNV vertex is an EWdoublet antineutrino or an EW-singlet antineutrino.

Within the context of our theoretical framework, our relation for decays of this type is

$$
(\tau / B)_{p \rightarrow \ell^{+} \nu \bar{\nu}} \sim\left(G_{F} m_{p}^{2}\right)^{-2}\left[\frac{R_{2}^{\left(\ell^{+} M\right)}}{\bar{R}_{3}^{\left(\ell^{+} \nu \bar{\nu}\right)}}\right](\tau / B)_{p \rightarrow \ell^{+} M} .
$$

Using the experimental lower bounds on $(\tau / B)_{p \rightarrow \ell^{+} \omega}$ given in Table I, we obtain the estimate $(\tau / B)_{p \rightarrow \ell^{+} \nu \bar{\nu}} \gtrsim 10^{45} \mathrm{yr}$ for $\ell^{+}=e^{+}, \mu^{+}$. This is much stronger than the lower bounds from direct searches, which are $(\tau / B)_{p \rightarrow e^{+} \nu \bar{\nu}}>$ $1.7 \times 10^{32} \mathrm{yr}$ and $(\tau / B)_{p \rightarrow \mu^{+} \nu \bar{\nu}}>2.2 \times 10^{32} \mathrm{yr}[18]$.
VII. $n \rightarrow \bar{\nu} \bar{\nu} \nu$

Finally, we consider the decays of the generic form $n \rightarrow \bar{\nu} \bar{\nu} \nu$. Graphs that contribute to these decays are shown in Fig. 8. In the processes depicted in Figs. 8(a) and 8(b), the $\bar{\nu}$ that emanates from the BNV four-fermion vertex can be either an EW-doublet antineutrino (of any flavor) or an EW-singlet antineutrino, while the $\nu \bar{\nu}$ pair produced by the virtual $Z$ are EW-doublet (anti)neutrinos. In Fig. 8(c), the two antineutrinos and the neutrino are all of EW-doublet type. We estimate

$$
(\tau / B)_{n \rightarrow \bar{\nu} \bar{\nu} \nu} \sim\left(G_{F} m_{n}^{2}\right)^{-2}\left[\left(2^{5} \pi^{2}\right)\left(1-\frac{m_{M}^{2}}{m_{n}^{2}}\right)\right](\tau / B)_{n \rightarrow \bar{\nu} M}
$$

Using the experimental lower limit $(\tau / B)_{n \rightarrow \bar{\nu} \omega}>1.08 \times$ $10^{32}$ yr [13], as listed in Table I, we obtain the estimate $(\tau / B)_{n \rightarrow \bar{\nu} \nu \bar{\nu}} \gtrsim 10^{44} \mathrm{yr}$. This is much stronger than the lower bound from a direct experimental search, namely

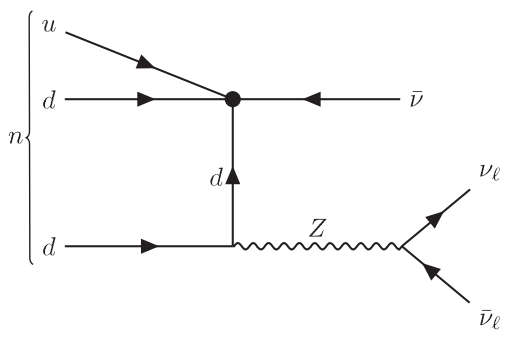

(a)

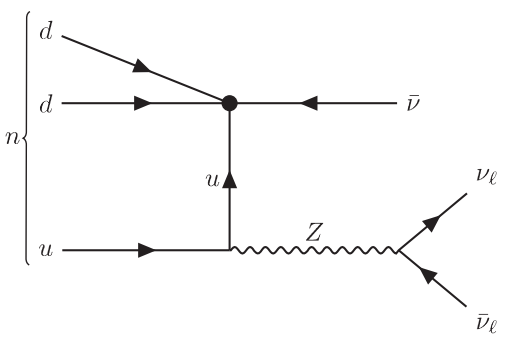

(b)

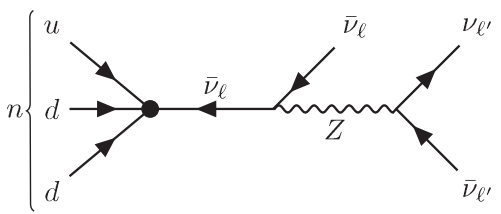

(c)

FIG. 8. Feynman diagrams for $n \rightarrow \bar{\nu} \bar{\nu} \nu$ decay. 
$(\tau / B)_{n \rightarrow \text { inv. }}>0.58 \times 10^{30} \mathrm{yr} \quad[19]$. This experimental lower bound was set by the KamLAND experiment from a search for gamma rays from the deexcitation of the ${ }^{11} \mathrm{C}$ nucleus that would result from the $n \rightarrow$ inv. decay of a neutron in a ${ }^{12} \mathrm{C}$ atom in its liquid scintillator detector.

\section{OTHER DECAY MODES}

Our method can also be applied to other nucleon decay modes, such as $p \rightarrow \ell^{+} \gamma \gamma$ and $n \rightarrow \bar{\nu} \gamma \gamma$, where here the $\gamma \gamma$ part of the final state are "continuum" photons, i.e., not photons that arise from a cascade decay such as $p \rightarrow \ell^{+} \pi^{0}$ followed by $\pi^{0} \rightarrow \gamma \gamma$ or $p \rightarrow \ell^{+} \eta$ followed by $\eta \rightarrow \gamma \gamma$. Graphs for these decays with continuum diphotons are obtained from those for $p \rightarrow \ell^{+} \gamma$ in Fig. 1 and for $n \rightarrow \bar{\nu} \gamma$ in Fig. 3 and hence are not shown separately. Using the same methods as before, we obtain

$$
(\tau / B)_{p \rightarrow \ell^{+} \gamma \gamma} \sim\left(4 \pi \alpha_{\mathrm{em}}\right)^{-2}\left[\frac{R_{2}^{\left(\ell^{+} M\right)}}{\bar{R}_{3}^{\left(\ell^{+} \gamma \gamma\right)}}\right](\tau / B)_{p \rightarrow \ell^{+} M}
$$

and

$$
\begin{aligned}
(\tau / B)_{n \rightarrow \bar{\nu} \gamma \gamma} & \sim\left(4 \pi \alpha_{\mathrm{em}}\right)^{-2}\left[\frac{R_{2}^{(\bar{\nu} M)}}{\bar{R}_{3}^{(\bar{\nu} \gamma \gamma)}}\right](\tau / B)_{n \rightarrow \bar{\nu} M} \\
& \sim\left[\left(2^{5} \pi^{2}\right)\left(1-\frac{m_{M}^{2}}{m_{n}^{2}}\right)\right](\tau / B)_{n \rightarrow \bar{\nu} M} .
\end{aligned}
$$

Using the experimental lower bounds on $p \rightarrow e^{+} M$ and $p \rightarrow \mu^{+} M$ listed for $M=\rho^{0}, \omega$ in Table I, the more stringent of which are for $p \rightarrow \ell^{+} \omega$, we obtain the estimates $(\tau / B)_{p \rightarrow e^{+} \gamma \gamma} \gtrsim 2 \times 10^{37} \mathrm{yr}$ and $(\tau / B)_{p \rightarrow \mu^{+} \gamma \gamma} \gtrsim$ $3 \times 10^{37} \mathrm{yr}$. An experimental lower bound from a direct search is $p \rightarrow e^{+} \gamma \gamma$ decay mode, namely $(\tau / B)_{p \rightarrow e^{+} \gamma \gamma}>$ $1.0 \times 10^{32} \mathrm{yr}$ [16]. Our estimated lower bound is much stronger than this direct limit. We are not aware of any published experimental lower bound on $(\tau / B)_{p \rightarrow \mu^{+} \gamma \gamma}$.

By similar methods, we obtain the estimated lower bound $(\tau / B)_{n \rightarrow \bar{\nu} \gamma \gamma} \gtrsim 10^{36} \mathrm{yr}$. An experimental lower bound is $(\tau / B)_{n \rightarrow \bar{\nu} \gamma \gamma}>2.39 \times 10^{32} \mathrm{yr}$ from the IMB3 experiment [13]. This was an inclusive search for any events of this type, which also allowed for the possibility that the invariant diphoton mass was equal to $m_{\pi^{0}}$ or $m_{\eta}$ to within the detector resolution [21]. Our estimated lower bound is again much stronger than this direct limit. These results are summarized in Table IV. One can also apply these techniques to relate other baryon-violating processes to each other. We have done this to derive improved bounds on certain $\Delta B=-2$ dinucleon decays. These results are reported elsewhere.

\section{CONCLUSIONS}

In this paper we have calculated estimated lower bounds on the partial lifetimes for several nucleon decays, including $p \rightarrow \ell^{+} \ell^{\prime+} \ell^{\prime-}, \quad n \rightarrow \bar{\nu} \ell^{+} \ell^{\prime-}, \quad p \rightarrow \ell^{+} \nu \bar{\nu}, \quad$ and $n \rightarrow \bar{\nu} \bar{\nu} \nu$, where $\ell$ and $\ell^{\prime}$ denote $e$ or $\mu$. We assume a minimal theoretical framework in which the only physics beyond the SM is that which produces the four-fermion operators in the baryon-number-violating effective Lagrangian responsible for these nucleon decays. Thus, if nucleon decays were to be observed with lower partial lifetimes than the bounds derived here, this would be of interest not only as evidence of baryon-number violation, but also as evidence of BNV mass scales lower than the GUT-type scales assumed in the minimal framework used here. Our method relies on relating the rates for these decay modes to the rates for decay modes of the form $p \rightarrow \ell^{+} M$ and $n \rightarrow \bar{\nu} M$, where $M$ is a pseudoscalar or vector meson, and then using the experimental lower bounds on these latter decays. Although our estimates are rough, our lower bounds are substantially stronger than lower bounds on the partial lifetimes for these decays from direct experimental searches. We also present corresponding estimated lower bounds on partial lifetimes for the radiative decays $p \rightarrow \ell^{+} \gamma, n \rightarrow \bar{\nu} \gamma, p \rightarrow \ell^{+} \gamma \gamma$, and $n \rightarrow \bar{\nu} \gamma \gamma$. There are strong motivations for pushing the search for nucleon decay to greater sensitivity in many channels. It is hoped that this search will be carried out with current data and with future nucleon decay experiments.

\section{ACKNOWLEDGMENTS}

This research was supported in part by the National Science Foundation Grants No. NSF-PHY-1620628 and No. NSF-PHY-1915093 (R. S.).

\section{APPENDIX: PHASE-SPACE FORMULAS}

In general, the decay rate of a parent particle $N$ with fourmomentum $p$ satisfying $p^{2}=m_{N}^{2}$ to a final state (f.s.) consisting of $n$ particles with four-momenta $p_{i}, 1 \leq i \leq n$, is given by

$$
\Gamma_{N \rightarrow f . s .}=\frac{S}{2 m_{N}} \int d R_{n}\left|A_{N \rightarrow f . s .}\right|^{2},
$$

where a sum over polarizations of final-state particles and an average over the polarizations of the parent particle are understood; $A_{N \rightarrow \text { f.s. }}$ denotes the amplitude for the decay; $S$ is a symmetry factor to take account of possible identical particles in the final state; and the integration over the $n$-body final-state phase space is given by 


$$
\int d R_{n}=\frac{1}{(2 \pi)^{3 n-4}} \int\left[\prod_{i=1}^{n} \frac{d^{3} p_{i}}{2 E_{i}}\right] \delta^{4}\left(p-\left(\sum_{i=1}^{n} p_{i}\right)\right) .
$$

For the nucleon decays of interest here we have $m_{N}$ with $N=p$ or $N=n$, and we denote $p_{i}^{2}=m_{i}^{2}$.

It is useful to consider the phase-space integration by itself, defining an $n$-body phase-space factor $R_{n}$ as

$$
R_{n}=\int d R_{n}
$$

For our applications, we will sometimes want to explicitly indicate the final-state particles, and for this purpose, we will use the notation $R_{n}^{(f . s .)}$. For example, for $p \rightarrow e^{+} \pi^{0}$, this phase-space factor is written as $R_{2}^{\left(e^{+} \pi^{0}\right)}$, and so forth for other decays.

The quantity $R_{n}$ has mass dimension $2(n-2)$, so we define a dimensionless phase-space factor

$$
\bar{R}_{n} \equiv\left(m_{N}\right)^{-2(n-2)} R_{n} .
$$

In general,

$$
R_{2}=\frac{1}{8 \pi}\left[\lambda\left(1, \delta_{1}, \delta_{2}\right)\right]^{1 / 2},
$$

where

$$
\lambda(x, y, z)=x^{2}+y^{2}+z^{2}-2(x y+y z+z x)
$$

and

$$
\delta_{i}=\left(\frac{m_{i}}{m_{N}}\right)^{2}
$$

In the case where $m_{i}^{2} / m_{N}^{2} \ll 1$ for all $i$, we denote the resultant $R_{n}$ as $R_{n, 0}$. A general formula is

$$
\bar{R}_{n, 0}=\frac{1}{2^{4 n-5} \pi^{2 n-3} \Gamma(n) \Gamma(n-1)} \quad \text { for } n \geq 2,
$$

where $\Gamma(n)$ is the Euler gamma function. In particular, for $n=2$ and $n=3$,

$$
R_{2,0}=\bar{R}_{2,0}=\frac{1}{2^{3} \pi}
$$

and

$$
\bar{R}_{3,0}=\frac{1}{2^{8} \pi^{3}} .
$$

For final states of nucleon decays in which an $e^{ \pm}$occurs, its mass satisfies the above condition of being negligibly small with respect to $m_{N}$. For leptonic final states involving one or two $\mu^{ \pm}$and a third particle of zero or negligibly small mass, we will make use of two formulas for $\bar{R}_{3}$. These follow from the general formula for the three-body phase space, $\bar{R}_{3}$ with one massless final-state particle, which is [22]

$$
\begin{aligned}
\left(2^{8} \pi^{3}\right) \bar{R}_{3}\left(m_{1}, m_{2}, 0\right)= & \left(1+\delta_{1}+\delta_{2}\right)\left[\lambda\left(1, \delta_{1}, \delta_{2}\right)\right]^{1 / 2}+2\left|\delta_{1}-\delta_{2}\right| \ln \left[\frac{\delta_{1}+\delta_{2}-\left(\delta_{1}-\delta_{2}\right)^{2}+\left|\delta_{1}-\delta_{2}\right|\left[\lambda\left(1, \delta_{1}, \delta_{2}\right)\right]^{1 / 2}}{2 \sqrt{\delta_{1} \delta_{2}}}\right] \\
& -2\left(\delta_{1}+\delta_{2}-2 \delta_{1} \delta_{2}\right) \ln \left[\frac{1-\delta_{1}-\delta_{2}+\left[\lambda\left(1, \delta_{1}, \delta_{2}\right)\right]^{1 / 2}}{2 \sqrt{\delta_{1} \delta_{2}}}\right] .
\end{aligned}
$$

Note that this is symmetric under the interchange $m_{1} \leftrightarrow m_{2}$ and thus $\delta_{1} \leftrightarrow \delta_{2}$.

The first special case of (A11) that we will need is for $m_{1}=m$, with $m_{2}$ and $m_{3}$ zero or negligibly small, so $\delta_{1}=\left(m / m_{N}\right)^{2} \equiv \delta$. This case applies for decays such as $p \rightarrow \mu^{+} e^{+} e^{-}$and $n \rightarrow \bar{\nu} \mu^{ \pm} e^{\mp}$. For this case we have

$$
\left(2^{8} \pi^{3}\right) \bar{R}_{3}(m, 0,0)=1-\delta^{2}-2 \delta \ln \left(\frac{1}{\delta}\right) .
$$

Numerically, the right-hand side of Eq. (A12) has the value 0.889 for $m=m_{\mu}$ with parent particle $p$ or $n$. The second special case of (A11) that we will need is for $m_{1}=m_{2}=m$, so $\delta_{1}=\delta_{2}=\left(m / m_{N}\right)^{2} \equiv \delta$. This case applies for decays such as $p \rightarrow e^{+} \mu^{+} \mu^{-}, n \rightarrow \bar{\nu} \mu^{+} \mu^{-}$. We have

$$
\begin{aligned}
& \left(2^{8} \pi^{3}\right) \bar{R}_{3}(m, m, 0) \\
& \quad=(1+2 \delta) \sqrt{1-4 \delta}-4 \delta(1-\delta) \ln \left[\frac{1-2 \delta+\sqrt{1-4 \delta}}{2 \delta}\right] .
\end{aligned}
$$

Numerically, the right-hand side of Eq. (A13) has the value 0.782 for $m=m_{\mu}$ with parent particle $p$ or $n$.

Since the form of $\mathcal{L}_{\text {eff }}$ in Eq. (7) depends on the unknown details of the baryon-number-violating BSM physics, a knowledge of this BSM physics would be necessary to calculate the full convolution of $|A|^{2}$ weighted with the three-body phase space in the $n=3$ case of Eq. (A1). This may be contrasted with the case in $\mu$ decay to massive neutrinos, where such calculations have been performed for both $V-A$ charged currents and general Lorentz structure [23]. 
[1] G. 't Hooft, Phys. Rev. Lett. 37, 8 (1976).

[2] H. Georgi and S. L. Glashow, Phys. Rev. Lett. 32, 438 (1974).

[3] H. Fritzsch and P. Minkowski, Ann. Phys. (N.Y.) 93, 193 (1975).

[4] P. Langacker, Phys. Rep. 72, 185 (1981); P. Nath and P. Fileviez-Perez, Phys. Rep. 441, 191 (2007).

[5] K. S. Babu, E. Kearns et al. (Snowmass 2013 Baryon Number Violation Group), arXiv:1311.5285.

[6] A. D. Sakharov, Zh. Eksp. Teor. Fiz. Pis'ma 5, 32 (1967) [JETP Lett. B 91, 24 (1967)].

[7] S. Weinberg, Phys. Rev. Lett. 43, 1566 (1979); Phys. Rev. D 22, 1694 (1980).

[8] F. Wilczek and A. Zee, Phys. Rev. Lett. 43, 1571 (1979).

[9] S. Aoki et al., Phys. Rev. D 62, 014506 (2000); Y. Aoki, T. Izubuchi, E. Shintani, and A. Soni, Phys. Rev. D 96, 014506 (2017), and references therein.

[10] K. Abe et al. (SK Collaboration), Phys. Rev. D 95, 012004 (2017).

[11] K. Abe et al. (SK Collaboration), Phys. Rev. D 96, 012003 (2017).

[12] K. Abe et al. (SK Collaboration), Phys. Rev. Lett. 113, 121802 (2014).
[13] C. McGrew et al. (IMB-3 Collaboration), Phys. Rev. D 59, 052004 (1999).

[14] S. Seidel et al. (IMB Collaboration), Phys. Rev. Lett. 61, 2522 (1988).

[15] S. Sussman et al. (SK Collaboration), arXiv:1811.12430.

[16] C. Berger et al. (Fréjus Collaboration), Phys. Lett. B 269, 227 (1991).

[17] V. Takhistov et al. (SK Collaboration), Phys. Rev. Lett. 115, 121803 (2015).

[18] V. Takhistov et al. (SK Collaboration), Phys. Rev. Lett. 113, 101801 (2014).

[19] T. Araki et al. (KamLAND Collaboration), Phys. Rev. Lett. 96, 101802 (2006).

[20] T. Hambye and J. Heeck, Phys. Rev. Lett. 120, 171801 (2018).

[21] C. McGrew (private communication). We thank C. McGrew for a helpful discussion concerning Ref. [13].

[22] E. Byckling and K. Kajantie, Particle Kinematics (Wiley, New York, 1973), p. 111.

[23] R. E. Shrock, Phys. Rev. D 24, 1275 (1981); Phys. Lett. B 112, 382 (1982). 